\title{
Dunkl-Type Operators with Projection Terms Associated to Orthogonal Subsystems in Root System
}

\author{
Fethi BOUZEFFOUR
}

Department of Mathematics, King Saudi University, College of Sciences, P.O. Box 2455 Riyadh 11451, Saudi Arabia

E-mail: fbouzaffour@ksu.edu.sa

Received April 24, 2013, in final form October 16, 2013; Published online October 23, 2013

http://dx.doi.org/10.3842/SIGMA.2013.064

\begin{abstract}
In this paper, we introduce a new differential-difference operator $T_{\xi}\left(\xi \in \mathbb{R}^{N}\right)$ by using projections associated to orthogonal subsystems in root systems. Similarly to Dunkl theory, we show that these operators commute and we construct an intertwining operator between $T_{\xi}$ and the directional derivative $\partial_{\xi}$. In the case of one variable, we prove that the Kummer functions are eigenfunctions of this operator.
\end{abstract}

Key words: special functions; differential-difference operators; integral transforms

2010 Mathematics Subject Classification: 33C15; 33D52; 35A22

\section{Introduction}

In a series of papers $[3,4,5,6]$, C.F. Dunkl builds up the framework for a theory of differentialdifference operators and special functions related to root systems. Beside them, there are now various further Dunkl-type operators, in particular the trigonometric Dunkl operators of Heckman [7, 8], Opdam [14], Cherednik [2], and the important q-analogues of Macdonald and Cherednik [13], see also $[1,11]$.

The main objective of this paper is to present a new class of differential-difference operators $T_{\xi}, \xi \in \mathbb{R}^{N}$ with the help of orthogonal projections related to orthogonal subsystems in root systems. In other words, our operators follow from Dunkl operator after replacing the usual reflections that exist in the definition of the operator with their corresponding orthogonal projections. Several problems related to the Dunkl theory arise in the setting of our operators, in particular, commutativity of $\left\{T_{\xi}, \xi \in \mathbb{R}^{N}\right\}$ and the existence of the intertwining operators.

The outline of the content of this paper is as follows. In Section 2, we collect some definitions and results related to root systems and Dunkl operators which will be relevant for the sequel. In Section 3, we introduce new differential-difference operators $T_{\xi}$ and we prove the first main result. In Section 4, we give an explicit formula for the intertwining operator between $T_{\xi}$ and the directional derivative. In Section 5, we study the one variable case. Finally, in Section 6 we study the cases of orthogonal subsets in root systems of type $A_{N-1}$ and $B_{N}$.

\section{Dunkl operators}

Let us begin to recall some results concerning the root systems and Dunkl operators. A useful reference for this topic is the book by Humphreys [9]. Let $\alpha \in \mathbb{R}^{N} \backslash\{0\}$, we denote by $s_{\alpha}$ the 
reflection onto the hyperplane orthogonal to $\alpha$; that is,

$$
s_{\alpha}(x)=x-2 \frac{\langle x, \alpha\rangle}{|\alpha|^{2}} \alpha,
$$

where $\langle\cdot, \cdot\rangle$ denotes the Euclidean scalar product on $\mathbb{R}^{N}$, and $|x|=\sqrt{\langle x, x\rangle}$.

A root system is a finite set $R$ of nonzero vectors in $\mathbb{R}^{N}$ such that for any $\alpha \in R$ one has

$$
s_{\alpha}(R)=R, \quad \text { and } \quad R \cap \mathbb{R} \alpha=\{ \pm \alpha\} .
$$

A positive subsystem $R_{+}$is any subset of $R$ satisfying $R=R_{+} \cup\left\{-R_{+}\right\}$. The Weyl group $W=W(R)$ (or real finite reflection group) generated by the root system $R \subset \mathbb{R}^{N}$ is the subgroup of orthogonal group $O(N)$ generated by $\left\{s_{\alpha}, \alpha \in R\right\}$. A multiplicity function on $R$ is a complex-valued function $\kappa: R \rightarrow \mathbb{C}$ which is invariant under the Weyl group $W$, i.e.,

$$
\kappa(\alpha)=\kappa(g \alpha), \quad \forall \alpha \in R, \quad \forall g \in W .
$$

Let $\xi \in \mathbb{R}^{N}$, the Dunkl operator $\mathcal{D}_{\xi}$ associated with the Weyl group $W(R)$ and the multiplicity function $\kappa$, is the first order differential-difference operator:

$$
\left(\mathcal{D}_{\xi} f\right)(x)=\partial_{\xi} f(x)+\sum_{\alpha \in R_{+}} \kappa(\alpha)\langle\alpha, \xi\rangle \frac{f(x)-f\left(s_{\alpha} x\right)}{\langle x, \alpha\rangle} .
$$

Here $\partial_{\xi}$ is the direction derivative corresponding to $\xi$ and $s_{\alpha}$ is the orthogonal reflection onto the hyperplane orthogonal to $\alpha$.

The Dunkl operator $\mathcal{D}_{\xi}$ is a homogeneous differential-difference operator of degree -1 . By the $W$-invariance of the multiplicity function $\kappa$, we have

$$
g^{-1} \circ \mathcal{D}_{\xi} \circ g=\mathcal{D}_{g \xi}, \quad \forall g \in W(R), \quad \xi \in \mathbb{R}^{N} .
$$

The remarkable property of the Dunkl operators is that the family $\left\{\mathcal{D}_{\xi}, \xi \in \mathbb{R}^{N}\right\}$ generates a commutative algebra of linear operators on the $\mathbb{C}$-algebra of polynomial functions.

\section{Operators of Dunkl-type}

Let $R$ be a root system. A subset $R^{\prime}$ of $R$ is called a subsystem of $R$ if it satisfies the following conditions:

i) If $\alpha \in R^{\prime}$, then $-\alpha \in R^{\prime}$;

ii) If $\alpha, \beta \in R^{\prime}$ and $\alpha+\beta \in R$, then $\alpha+\beta \in R^{\prime}$.

A subsystem $R^{\prime}$ of a root system $R$ in $\mathbb{R}^{N}$ consisting of pairwise orthogonal roots is called orthogonal subsystem. In this case the related Weyl group $W\left(R^{\prime}\right)$ is a subgroup of $\mathbb{Z}_{2}^{N}$. For a vector $\alpha \in \mathbb{R}^{N} \backslash\{0\}$, we write

$$
\tau_{\alpha}(x)=x-\frac{\langle x, \alpha\rangle}{|\alpha|^{2}} \alpha, \quad x \in \mathbb{R}^{N},
$$

for the orthogonal projection onto the hyperplane $(\mathbb{R} \alpha)^{\perp}=\{x,\langle x, \alpha\rangle=0\}$, so that the reflection $s_{\alpha}$ with respect to hyperplane orthogonal to $\alpha$ is related to $\tau_{\alpha}$ by

$$
\tau_{\alpha}=\frac{1}{2}\left(1+s_{\alpha}\right)
$$


The hyperplane $(\mathbb{R} \alpha)^{\perp}$ is the invariant set of $\tau_{\alpha}$. If $\langle\alpha, \beta\rangle=0$, then the orthogonal projections $\tau_{\alpha}$ and $\tau_{\beta}$ commute. The conjugate of orthogonal projection onto a hyperplane is again an orthogonal projection onto a hyperplane: suppose $u \in O(N)$ and $\alpha \in \mathbb{R}^{N} \backslash\{0\}$ then

$$
u \tau_{\alpha} u^{-1}=\tau_{u \alpha} .
$$

Let $R$ be a root system and $R^{\prime}$ a positive orthogonal subsystem of $R$. For $\xi \in \mathbb{R}^{N}$, we define the differential-difference operator $T_{\xi}$ by

$$
\left(T_{\xi} f\right)(x)=\partial_{\xi} f(x)+\sum_{\alpha \in R^{\prime}} \kappa(\alpha)\langle\alpha, \xi\rangle \frac{f(x)-f\left(\tau_{\alpha} x\right)}{\langle x, \alpha\rangle} .
$$

where $\kappa$ is a multiplicity function on $R^{\prime}$. For $j=1, \ldots, N$ denotes $T_{e_{j}}$ by $T_{j}$. The operator $T_{\xi}$ can be considered as a deformation of the usual directional derivatives and when $\kappa=0$, the operator $T_{\xi}$ reduces to the corresponding directional derivative. Furthermore, there is overlap between the notations (2) and (1). In fact, the operator (2) follows from Dunkl operator after replacing the reflections terms that exist in (1) by orthogonal projection terms.

Example 1. In the rank-one case, the root system is of type $A_{1}$ and the corresponding reflection $s$ and orthogonal projection $\tau$ are given by

$$
s(x)=-x, \quad \tau(x)=\frac{1}{2}(1+s)(x)=0 .
$$

The Dunkl-type operator $T_{\kappa}$ associated with the projection $\tau$ and the multiplicity parameters $\kappa$ $(\kappa \in \mathbb{C})$ is given by

$$
T_{\kappa} f(x)=f^{\prime}(x)+\kappa \frac{f(x)-f(\tau(x))}{x}=f^{\prime}(x)+\kappa \frac{f(x)-f(0)}{x} .
$$

Example 2. Let $R=\left\{ \pm\left(e_{1} \pm e_{2}\right), \pm e_{1}, \pm e_{2}\right\}$ be a root system of type $B_{2}$ in the 2-plane and $R^{\prime}=\left\{e_{1} \pm e_{2}\right\}$ be a positive orthogonal subsystem in $R$. The related Dunkl-type operators to $R^{\prime}$ and to the positive parameters $\left(\kappa_{1}, \kappa_{2}\right)$ are given by

$$
\begin{aligned}
& T_{1}=\partial_{x}+\kappa_{1} \frac{f(x, y)-f((x+y) / 2,(x+y) / 2)}{x-y}+\kappa_{2} \frac{f(x, y)-f((x-y) / 2,(x-y) / 2)}{x+y}, \\
& T_{2}=\partial_{y}-\kappa_{1} \frac{f(x, y)-f((x+y) / 2,(x+y) / 2)}{x-y}+\kappa_{2} \frac{f(x, y)-f((x-y) / 2,(x-y) / 2)}{x+y} .
\end{aligned}
$$

We denote by $\Pi^{N}$ the space of polynomials and by $\Pi_{n}^{N}$ the subspace of homogenous polynomials of degree $n$.

Let $R^{\prime}=\left\{\alpha_{1}, \ldots, \alpha_{n}\right\}$ be a positive orthogonal subsystem of a root system $R$. Consider the operator $\rho_{i}$ defined on $\Pi^{N}$ by

$$
\left(\rho_{i} f\right)(x)=\frac{f(x)-f\left(\tau_{\alpha_{i}} x\right)}{\langle x, \alpha\rangle}, \quad i=1, \ldots, n .
$$

It follows from the equality

$$
\left(\rho_{j} f\right)(x)=-\frac{1}{\left|\alpha_{j}\right|^{2}} \int_{0}^{1} \partial_{\alpha_{j}} f\left(x-t \frac{\left\langle x, \alpha_{j}\right\rangle}{\left|\alpha_{j}\right|^{2}} \alpha_{j}\right) \mathrm{d} t
$$

that $T_{\xi}$ is a homogeneous operator of degree -1 on $\Pi^{N}$, that is, $T_{\xi} f \in \Pi_{n-1}^{N}$, for $f \in \Pi_{n}^{N}$, and leaves $\mathcal{S}(\mathbb{R})(\mathcal{S}(\mathbb{R})$ is the Schwartz space of rapidly decreasing functions on $\mathbb{R})$ invariant. 
Proposition 1. The operators $\rho_{i}(i=1, \ldots, n)$ have the following properties:

i) for $i, j=1, \ldots, n$, we have $\left[\rho_{i}, \rho_{j}\right]=0$;

ii) if $\alpha$ is an orthogonal vector to $\alpha_{i}$, then $\left[\partial_{\alpha}, \rho_{i}\right]=0$, where the commutator of two operators $A, B$ is defined by $[A, B]:=A B-B A$.

The family $\left\{\alpha_{1}, \ldots, \alpha_{n}\right\}$ is orthogonal, then there exist scalars $\xi_{1}, \ldots, \xi_{n}$ and a vector $\widehat{\xi} \in \mathbb{R}^{N}$ orthogonal to the subspace $\mathbb{R} \alpha_{1} \oplus \cdots \oplus \mathbb{R} \alpha_{n}$ such that

$$
\xi=\sum_{i=1}^{n} \xi_{i} \alpha_{i}+\widehat{\xi}
$$

This allows us to decompose the operator $T_{\xi}(2)$ associated with $R^{\prime}$ and the multiplicity parameters $\left(\kappa_{1}, \ldots, \kappa_{n}\right)$ in a unique way in the form

$$
T_{\xi}=\sum_{i=1}^{n} \xi_{i} T_{\alpha_{i}}+\partial_{\widehat{\xi}}
$$

We now have all ingredients to state and prove the first main result of the paper.

Theorem 1. Let $\xi, \eta \in \mathbb{R}^{N}$, then $\left[T_{\xi}, T_{\eta}\right]=0$.

Proof. A straightforward computation yields

$$
\left[T_{\xi}, T_{\eta}\right]=\sum_{i, j=1}^{n} \xi_{i} \eta_{j}\left[T_{\alpha_{i}}, T_{\alpha_{j}}\right]+\left[\partial_{\widehat{\xi}}, \partial_{\widehat{\eta}}\right]+\sum_{i=1}^{n} \xi_{i}\left[T_{\alpha_{i}}, \partial_{\widehat{\eta}}\right]-\eta_{i}\left[T_{\alpha_{i}}, \partial_{\widehat{\xi}}\right] .
$$

On the other hand,

$$
\begin{aligned}
{\left[T_{\alpha_{i}}, T_{\alpha_{j}}\right] } & =\left[\partial_{\alpha_{i}}+\kappa_{i}\left\|\alpha_{i}\right\| \rho_{i}, \partial_{\alpha_{j}}+\kappa_{j}\left\|\alpha_{j}\right\| \rho_{j}\right] \\
& =\left[\partial_{\alpha_{i}}, \partial_{\alpha_{j}}\right]+\kappa_{j}\left\|\alpha_{j}\right\|\left[\partial_{\alpha_{i}}, \rho_{j}\right]-\kappa_{i}\left\|\alpha_{i}\right\|\left[\partial_{\alpha_{j}}, \rho_{i}\right]+\kappa_{i} \kappa_{j}\left\|\alpha_{i}\right\|\left\|\alpha_{j}\right\|\left[\rho_{i}, \rho_{j}\right]
\end{aligned}
$$

and

$$
\left[T_{\alpha_{i}}, \partial_{\widehat{\xi}}\right]=\left[\partial_{\alpha_{i}}, \partial_{\widehat{\xi}}\right]+\kappa_{i}\left\|\alpha_{i}\right\|\left[\rho_{i}, \partial_{\xi}\right]
$$

From Proposition 1, we get

$$
\left[T_{\alpha_{i}}, T_{\alpha_{j}}\right]=0 \quad \text { and } \quad\left[T_{\alpha_{i}}, \partial_{\widehat{\xi}}\right]=0
$$

This proves the result.

One important consequence of the Theorem 1 , is that the operators $T_{\alpha_{1}}, \ldots, T_{\alpha_{m}}$ generate a commutative algebra.

\section{Intertwining operator}

In this section, we give an intertwining operator between $T_{\xi}$ and the directional derivative $\partial_{\xi}$. Consider a positive orthogonal subsystem $R^{\prime}=\left\{\alpha_{1}, \ldots, \alpha_{n}\right\}$ composed of $n$ vectors in a root system $R$, and $\kappa=\left(\kappa_{1}, \ldots, \kappa_{n}\right) \in \mathbb{C}^{n}$ and $\xi \in \mathbb{R}^{N}$. The associated Dunkl-type operator $T_{\xi}$ with $R^{\prime}$ and $\kappa$ takes the form

$$
\left(T_{\xi} f\right)(x)=\partial_{\xi} f(x)+\sum_{j=1}^{n} \kappa_{j}\left\langle\alpha_{j}, \xi\right\rangle \frac{f(x)-f\left(\tau_{\alpha_{j}} x\right)}{\left\langle x, \alpha_{j}\right\rangle} .
$$


Let $h: \mathbb{R}^{n} \times \mathbb{R}^{N} \rightarrow \mathbb{R}^{N}$ be the function defined by

$$
h(t, x)=x+\sum_{j=1}^{n}\left(t_{j}-1\right) \frac{\left\langle x, \alpha_{j}\right\rangle}{\left|\alpha_{j}\right|^{2}} \alpha_{j},
$$

where $t=\left(t_{1}, \ldots, t_{n}\right) \in \mathbb{R}^{n}$ and $x \in \mathbb{R}^{N}$.

We define

$$
\chi_{\kappa}(f)(x)=\frac{1}{\Gamma(\kappa)} \int_{[0,1]^{n}} f(h(t, x)) w(t) \mathrm{d} t,
$$

where $w(t)=\prod_{j=1}^{n}\left(1-t_{j}\right)^{\kappa_{j}-1}$ and $\Gamma(\kappa)=\prod_{j=1}^{n} \Gamma\left(\kappa_{j}\right)$.

Theorem 2. Let $f \in C^{\infty}\left(\mathbb{R}^{N}\right)$, then we have

$$
T_{\xi} \circ \chi_{\kappa} f(x)=\chi_{\kappa} \circ \partial_{\xi} f(x) \text {. }
$$

Proof. For $j=1, \ldots, n$, we denote by $\theta_{j}$ the orthogonal projection in $\mathbb{R}^{n}$ with respect to the hyperplane $\left(\mathbb{R} e_{j}\right)^{\perp}$ orthogonal to the vector $e_{j}$ of the canonical basis $\left(e_{1}, \ldots, e_{n}\right)$ of $\mathbb{R}^{n}$. The orthogonal projection $\theta_{j}$ acts on $\mathbb{R}^{n}$ as

$$
\theta_{j}(t)=\left(t_{1}, \ldots, t_{j-1}, 0, t_{j+1}, \ldots, t_{n}\right) .
$$

The system $R$ is orthogonal, then for $j=1, \ldots, n$, we have

$$
\begin{aligned}
h\left(t, \tau_{\alpha_{j}} x\right) & =\tau_{\alpha_{j}} x+\sum_{k=1}^{n}\left(t_{k}-1\right) \frac{\left\langle\tau_{\alpha_{j}} x, \alpha_{k}\right\rangle}{\left|\alpha_{k}\right|^{2}} \alpha_{k} \\
& =x-\frac{\left\langle x, \alpha_{j}\right\rangle}{\left|\alpha_{j}\right|^{2}} \alpha_{j}+\sum_{k=1, k \neq j}^{n}\left(t_{k}-1\right) \frac{\left\langle x, \alpha_{k}\right\rangle}{\left|\alpha_{k}\right|^{2}} \alpha_{k}=h\left(\theta_{j} t, x\right) .
\end{aligned}
$$

Let $f \in C^{\infty}\left(\mathbb{R}^{N}\right)$ and $\xi \in \mathbb{R}^{N}$. The mapping $x \rightarrow h(t, x)$ is linear on $\mathbb{R}^{N}$, then we can write

$$
\partial_{\xi}(f(h(t, x)))=\partial_{h(t, \xi)} f(h(t, x))=\partial_{\xi} f(h(t, x))+\sum_{j=1}^{n}\left(t_{j}-1\right) \frac{\left\langle\xi, \alpha_{j}\right\rangle}{\left|\alpha_{j}\right|^{2}} \partial_{\alpha_{j}} f(h(t, x)) .
$$

Hence,

$$
\begin{aligned}
\partial_{\xi} \chi_{\kappa}(f)(x)= & \frac{1}{\Gamma(\kappa)} \int_{[0,1]^{n}} \partial_{\xi}(f(h(t, x))) w(t) \mathrm{d} t=\frac{1}{\Gamma(\kappa)} \int_{[0,1]^{n}} \partial_{\xi} f(h(t, x)) w(t) \mathrm{d} t \\
& +\frac{1}{\Gamma(\kappa)} \sum_{j=1}^{n} \frac{\left\langle\xi, \alpha_{j}\right\rangle}{\left|\alpha_{j}\right|^{2}} \int_{[0,1]^{n}}\left(t_{j}-1\right) \partial_{\alpha_{j}} f(h(t, x)) w(t) \mathrm{d} t .
\end{aligned}
$$

Since we can write

$$
\partial_{t_{j}} f(h(t, x))=\frac{\left\langle x, \alpha_{k}\right\rangle}{\left|\alpha_{k}\right|^{2}} \partial_{\alpha_{j}} f(h(t, x))
$$

and

$$
\int_{0}^{1}\left(1-t_{j}\right)^{\kappa_{j}} \partial_{t_{j}} f(h(t, x)) \mathrm{d} t=-f\left(h\left(\theta_{j}(t), x\right)\right)+\kappa_{j} \int_{0}^{1}\left(1-t_{j}\right)^{\kappa_{j}-1} f(h(t, x)) \mathrm{d} t,
$$


we are lead to

$$
\begin{aligned}
\int_{[0,1]^{n}} & \partial_{\alpha_{j}} f(h(t, x))\left(t_{j}-1\right) w(t) \mathrm{d} t=\frac{\left|\alpha_{j}\right|^{2}}{\left\langle x, \alpha_{j}\right\rangle} \int_{[0,1]^{n}} \partial_{t_{j}} f(h(t, x))\left(t_{j}-1\right) w(t) \mathrm{d} t \\
= & \kappa_{j} \frac{\left|\alpha_{j}\right|^{2}}{\left\langle x, \alpha_{j}\right\rangle} \int_{[0,1]^{n}}\left(f\left(h\left(\theta_{j}(t), x\right)\right)-f(h(t, x))\right) w(t) \mathrm{d} t \\
= & -\kappa_{j} \Gamma(\kappa) \frac{\left|\alpha_{j}\right|^{2}}{\left\langle x, \alpha_{j}\right\rangle}\left(\chi_{\kappa}(f)(x)-\chi_{\kappa}(f)\left(\tau_{\alpha_{j}} x\right)\right) .
\end{aligned}
$$

This, combined with the last expression of $\partial_{\xi}\left(\chi_{\kappa} f\right)(x)$, yields

$$
\partial_{\xi} \chi_{\kappa}(f)(x)=\chi_{\kappa}\left(\partial_{\xi} f\right)(x)-\sum_{j=1}^{n} \kappa_{j}\left\langle\xi, \alpha_{j}\right\rangle \frac{\chi_{\kappa}(f)(x)-\chi_{\kappa}(f)\left(\tau_{j} x\right)}{\left\langle x, \alpha_{j}\right\rangle} .
$$

Therefore,

$$
T_{\xi}\left(\chi_{\kappa} f\right)(x)=\chi_{\kappa}\left(\partial_{\xi} f\right)(x) .
$$

\section{The one variable case}

The specialization of this theory to the one variable case has its own interest, because everything can be done there in a much more explicit way and new results for special functions in one variable can be obtained. In this setting there is only one Dunkl-type operator $T_{\kappa}$ associated up to scaling and it equals to

$$
T_{\kappa} f(x)=f^{\prime}(x)+\kappa \frac{f(x)-f(0)}{x} .
$$

This operator leaves the space of polynomials invariant and acts on the monomials as

$$
T_{\kappa} 1=0, \quad T_{\kappa} x^{n}=(n+\kappa) x^{n-1}, \quad n=1,2, \ldots
$$

Its square is given by

$$
T_{\kappa}^{2} f(x)=f^{\prime \prime}(x)+\frac{2 \kappa}{x} f^{\prime}(x)+\kappa(\kappa-1) \frac{f(x)-f(0)}{x^{2}}-\frac{\kappa(\kappa+1)}{x} f^{\prime}(0) .
$$

Consider the confluent hypergeometric function (see $[15, \S 7.1]$ )

$$
M(a, b ; z)=\sum_{n=0}^{\infty} \frac{(a)_{n}}{(b)_{n}} \frac{z^{n}}{n !},
$$

where $(a)_{n}$ is the Pochhammer symbol defined by

$$
(a)_{n}=\frac{\Gamma(a+n)}{\Gamma(a)} .
$$

This is a solution of the confluent hypergeometric differential equation

$$
z y^{\prime \prime}(z)+(b-z) y^{\prime}(z)=a y(z) .
$$

This function possesses the following Poisson integral representation (see $[15, \S 7.1]$ )

$$
M(a, b ; z)=\frac{\Gamma(b)}{\Gamma(a) \Gamma(b-a)} \int_{0}^{1} t^{a-1}(1-t)^{b-a-1} e^{z t} \mathrm{~d} t, \quad \Re(b)>\Re(a)>0 .
$$


Theorem 3. For $\lambda \in \mathbb{C}$ and $\kappa>-1$, the problem

$$
T_{\kappa} f(x)=i \lambda f(x), \quad f(0)=1,
$$

has a unique analytic solution $M_{\kappa}(i \lambda x)$ given by

$$
M_{\kappa}(i \lambda x)=M(1, \kappa+1 ; i \lambda x) .
$$

Proof. Searching a solution of (6) in the form $f(z)=\sum_{n=0}^{\infty} a_{n} x^{n}$. Replacing in (6), we obtain

$$
\sum_{n=0}^{\infty}(n+1+\kappa) a_{n+1} x^{n}=i \lambda \sum_{n=0}^{\infty} a_{n} x^{n} .
$$

Thus,

$$
a_{n+1}=\frac{i \lambda}{n+1+\kappa} a_{n} \quad \text { and } \quad a_{n}=\frac{(i \lambda)^{n}}{(\kappa+1)_{n}} .
$$

Remark 1. Multiply the equation (6) by $x$ and differentiating both sides, we see that a function $u$ of class $C^{2}$ on $\mathbb{R}$, is a solution of the equation (6), if and only if, it is a solution of the generalized eigenvalue problem

$$
x u^{\prime \prime}+(\kappa+1) u^{\prime}=i \lambda\left(x u^{\prime}+u\right) .
$$

Proposition 2. The function $\mathbf{M}_{\kappa}(z)$ defined by

$$
\mathbf{M}_{\kappa}(z)=\frac{M_{\kappa}(z)}{\Gamma(\kappa+1)}=\sum_{n=0}^{\infty} \frac{z^{n}}{\Gamma(\kappa+1+n)}
$$

satisfies the following properties:

(i) $\mathbf{M}_{\kappa}(z)$ is analytic in $\kappa$ and $z$;

(ii) $\mathbf{M}_{0}(z)=e^{z}$;

(iii) for $\Re(\kappa)>0$, the function $\mathbf{M}_{\kappa}(z)$, possesses the integral representation

$$
\mathbf{M}_{\kappa}(z)=\frac{1}{\Gamma(\kappa)} \int_{0}^{1}(1-t)^{\kappa-1} e^{z t} \mathrm{~d} t
$$

(iv) for $\Re(\kappa)>0$, we have

$$
\left|\mathbf{M}_{\kappa}^{(n)}(z)\right| \leq|z|^{n} e^{\Re(z)}, \quad n \in \mathbb{N}, \quad z \in \mathbb{C},
$$

in particular,

$$
\left|\mathbf{M}_{\kappa}(i \lambda x)\right| \leq 1, \quad \lambda, x \in \mathbb{R}
$$

(v) for $\Re(\kappa)>0$, and all $x \in \mathbb{R}^{*}$,

$$
\lim _{\lambda \rightarrow+\infty} \mathbf{M}_{\kappa}(i \lambda x)=0 .
$$


Proof. (i) and (ii) are immediate. (iii) follows from (5). For $n \in \mathbb{N}$, we have

$$
\mathbf{M}_{\kappa}^{(n)}(z)=\frac{z^{n}}{\Gamma(\kappa)} \int_{0}^{1}(1-t)^{\kappa} t^{n} e^{z t} \mathrm{~d} t
$$

So we find

$$
\left|\mathbf{M}_{\kappa}^{(n)}(z)\right| \leq \frac{|z|^{n}}{\Gamma(\kappa)} \int_{0}^{1}(1-t)^{\kappa} e^{\Re(z) t} \mathrm{~d} t \leq|z|^{n} e^{\Re(z)} .
$$

This proves (iv). (v) follows from (iii) and the Riemann-Lebesgue lemma.

Definition 1. We define the Kummer transform on $L^{1}(\mathbb{R})$ by

$$
\forall \lambda \in \mathbb{R}, \quad \mathcal{F}_{\kappa}(f)(\lambda)=\int_{\mathbb{R}} f(x) \mathbf{M}_{\kappa}(i \lambda x)(x) \mathrm{d} x .
$$

When $\kappa=0$, the transformation $\mathcal{F}_{0}$ reduces to the usual Fourier transform $\mathcal{F}$ that is given by

$$
\mathcal{F}(f)(\lambda)=\int_{\mathbb{R}} f(x) e^{i \lambda x} \mathrm{~d} x
$$

Theorem 4. Let $f$ be a function in $L^{1}(\mathbb{R})$ then $\mathcal{F}_{\kappa}(f)$ belongs to $C_{0}(\mathbb{R})$, where $C_{0}(\mathbb{R})$ is the space of continuous functions having zero as limit at the infinity. Furthermore,

$$
\left\|\mathcal{F}_{\kappa}(f)\right\|_{\infty} \leq\|f\|_{1} .
$$

Proof. It's clear that $\mathcal{F}_{\kappa}(f)$ is a continuous function on $\mathbb{R}$. From Proposition 2, we get for all $x \in \mathbb{R}^{*}$,

$$
\lim _{\lambda \rightarrow \infty} f(x) \mathbf{M}_{\kappa}(i \lambda x)=0 \quad \text { and } \quad\left|f(x) \mathbf{M}_{\kappa}(i \lambda x)\right| \leq|f(x)| .
$$

Since $f$ is in $L^{1}(\mathbb{R})$, we conclude by using the dominated convergence theorem that $\mathcal{F}_{\kappa}(f)$ belongs to $C_{0}(\mathbb{R})$ and

$$
\left\|\mathcal{F}_{\kappa}(f)\right\|_{\infty} \leq\|f\|_{1} .
$$

We now turn to exhibit a relationship between the Kummer transform and the Fourier transform. The crucial idea is to use the intertwining operator $\chi_{\kappa}$. We denote by $C^{\infty}(\mathbb{R})$ the space of infinitely differentiable functions $f$ on $\mathbb{R}$, provided with the topology defined by the semi norms

$$
\|f\|_{n, a}=\sup _{\substack{0 \leq k \leq n \\ x \in[-a, a]}}\left|f^{(k)}(x)\right|, \quad a>0, \quad n \in \mathbb{N} .
$$

In the rank-one case the intertwining operator (3) becomes

$$
\left(\chi_{\kappa} f\right)(x)=\frac{1}{\Gamma(\kappa)} \int_{0}^{1}(1-t)^{\kappa-1} f(t x) \mathrm{d} t .
$$

This operator is a particular case of the so called Erdélyi-Kober fractional integral $I^{\gamma, \delta}$, which is given by (see [10])

$$
\left(I^{\gamma, \delta} f\right)(x)=\frac{1}{\Gamma(\delta)} \int_{0}^{1}(1-t)^{\delta-1} t^{\gamma} f(t x) \mathrm{d} t, \quad \delta>0, \quad \gamma \in \mathbb{R} .
$$


It was shown in $[12, \S 3]$, that the Erdélyi-Kober fractional integral has a left-inverse

$$
D^{\gamma, \delta} I^{\gamma, \delta} f=f, \quad f \in C^{\infty}(\mathbb{R}),
$$

where

$$
D^{\gamma, \delta}=\prod_{k=1}^{n}\left(\gamma+k+x \frac{\mathrm{d}}{\mathrm{d} x}\right) I^{\gamma+\delta, n-\delta},
$$

and $n=\lceil\delta\rceil(\lceil\delta\rceil$ denotes the ceiling function the smallest integer $\geq \delta)$.

As a consequence of Theorem 2, we deduce that the operator $\chi_{\kappa}$ (9) has the fundamental intertwining property

$$
T_{\kappa} \circ \chi_{\kappa}=\chi_{\kappa} \circ \frac{\mathrm{d}}{\mathrm{d} x} \text {. }
$$

We regard it as a second main result since it allows us to move from the complicated operator $T_{\kappa}$ defined in (4) to the simple derivative operator $\frac{\mathrm{d}}{\mathrm{d} x}$.

Theorem 5. Let $\kappa>0$, the operator $\chi_{\kappa}$ is a topological isomorphism from $C^{\infty}(\mathbb{R})$ onto itself and its inverse $\chi_{\kappa}^{-1}$ is given for all $f \in C^{\infty}(\mathbb{R})$ by

$$
\chi_{\kappa}^{-1} f(x)=D^{0, \kappa} f(x)=\prod_{j=1}^{n}\left(j+x \frac{\mathrm{d}}{\mathrm{d} x}\right)\left(I^{\kappa+1, n-\kappa} f\right)(x),
$$

where $n=\lceil\kappa\rceil$.

Proof. Let $a>0$ and $f \in C^{\infty}(\mathbb{R})$. For $x \in[0, a], t \in[0,1]$ and $l \in \mathbb{N}$, we have the following estimate

$$
\left|t^{l}(1-t)^{\kappa-1} f^{(l)}(x t)\right| \leq\|f\|_{l, a}(1-t)^{\kappa-1} \quad \text { and } \quad \int_{0}^{1}(1-t)^{\kappa-1} \mathrm{~d} t=\frac{1}{\kappa} .
$$

By the theorem of derivation under the integral sign, we can prove that

$$
\chi_{\kappa} f \in C^{\infty}(\mathbb{R}) \quad \text { and } \quad\left\|\chi_{\kappa}(f)\right\|_{l, a} \leq \frac{1}{\Gamma(\kappa+1)}\|f\|_{l, a} .
$$

Then $\chi_{\kappa}$ is a linear continuous mapping from $C^{\infty}(\mathbb{R})$ onto its self. From formula (10) the operator

$$
D^{0, \kappa}=\prod_{j=1}^{n}\left(j+x \frac{\mathrm{d}}{\mathrm{d} x}\right) \circ I^{\kappa+1, n-\kappa}
$$

is a left-inverse of $\chi_{\kappa}$. This shows that $\chi_{\kappa}$ is injective and $D^{0, \kappa}$ is surjective. So it suffices to prove that $D^{0, \kappa}$ is injective.

Let $f$ be a function in $C^{\infty}(\mathbb{R})$ such that $D^{0, \kappa} f=0$. Then the function $g=I^{\kappa+1, n-\kappa} f \in$ $C^{\infty}(\mathbb{R})$ is a solution of the linear differential equation

$$
\prod_{j=1}^{n}\left(1+j+x \frac{\mathrm{d}}{\mathrm{d} x}\right) y(x)=0 .
$$

Since, the last differential equation has a unique $C^{\infty}$-solution, which is equal to $y(x)=0$, it follows that $g=0$.

From (10) the operator $I^{\kappa+1, \kappa}$ has a left-inverse, then $f=0$. This shows that $\chi_{\kappa}$ is a bijective operator. 
Let $\kappa>0$, we define the dual intertwining operator ${ }^{t} \chi_{\kappa}$ on $\mathcal{D}(\mathbb{R})(\mathcal{D}(\mathbb{R})$ is the space of $C^{\infty}$-functions on $\mathbb{R}$ with compact support) by

$$
\left({ }^{t} \chi_{\kappa} f\right)(x)=\frac{1}{\Gamma(\kappa)} \int_{|x|}^{+\infty}(t-|x|)^{\kappa-1} t^{-\kappa} f(\operatorname{sgn}(x) t) \mathrm{d} t, \quad x \in \mathbb{R} \backslash\{0\} .
$$

Proposition 3. The operator ${ }^{t} \chi_{\kappa}$ is a topological automorphism of $\mathcal{D}(\mathbb{R})$, and satisfies the transmutation relation:

$$
\int_{\mathbb{R}}\left(\chi_{\kappa} f\right)(x) g(x) \mathrm{d} x=\int_{\mathbb{R}} f(x)\left({ }^{t} \chi_{\kappa} g\right)(x) \mathrm{d} x, \quad f \in C^{\infty}(\mathbb{R}) .
$$

Proof. Let $f \in C^{\infty}(\mathbb{R})$ and $g \in \mathcal{D}(\mathbb{R})$, we have

$$
\begin{aligned}
\int_{\mathbb{R}}\left(\chi_{\kappa} f\right)(x) g(x) \mathrm{d} x= & \frac{1}{\Gamma(\kappa)} \int_{0}^{+\infty} \int_{0}^{x}(x-t)^{\kappa-1} f(t) \mathrm{d} t g(x) x^{-\kappa} \mathrm{d} x \\
& -\frac{1}{\Gamma(\kappa)} \int_{0}^{\infty} \int_{0}^{x}(x-t)^{\kappa-1} f(-t) \mathrm{d} t g(-x) x^{-\kappa} \mathrm{d} x .
\end{aligned}
$$

Using Fubini's theorem and a change of variable, we get

$$
\begin{aligned}
\int_{\mathbb{R}}\left(\chi_{\kappa} f\right)(x) g(x) \mathrm{d} x= & \frac{1}{\Gamma(\kappa)} \int_{0}^{+\infty} \int_{t}^{\infty} x^{-\kappa}(x-t)^{\kappa-1} g(x) \mathrm{d} x f(t) \mathrm{d} t \\
& +\frac{1}{\Gamma(\kappa)} \int_{-\infty}^{0} \int_{-t}^{\infty} x^{-\kappa}(x+t)^{\kappa-1} g(-x) \mathrm{d} x f(t) \mathrm{d} t .
\end{aligned}
$$

Therefore,

$$
\begin{aligned}
\int_{\mathbb{R}}\left(\chi_{\kappa} f\right)(x) g(x) \mathrm{d} x & =\frac{1}{\Gamma(\kappa)} \int_{\mathbb{R}} \int_{|t|}^{\infty} x^{-\kappa}(x-|t|)^{\kappa-1} g(\operatorname{sign}(t) x) \mathrm{d} x f(t) \mathrm{d} t \\
& =\int_{\mathbb{R}} f(t)\left({ }^{t} \chi_{\kappa} g\right)(t) \mathrm{d} t .
\end{aligned}
$$

Proposition 4. Let $\kappa>0$, the Kummer transform $\mathcal{F}_{\kappa}$ satisfies the decomposition

$$
\mathcal{F}_{\kappa}(f)=\mathcal{F} \circ{ }^{t} \chi_{\kappa}(f), \quad f \in \mathcal{D}(\mathbb{R}) .
$$

Proof. The result follows from Proposition 3.

\section{Multivariable case}

\subsection{Direct product setting}

In this subsection, we consider the direct product of the one-dimensional models, which means that the Weyl group of the corresponding subsystem of root system is a subgroup of $\mathbb{Z}_{2}^{N}$.

We denote by $\tau_{k}$ (for each $k$ from 1 to $N$ ) the orthogonal projection with respect to the hyperplane orthogonal to $e_{k}$, that is to say for every $x=\left(x_{1}, \ldots, x_{N}\right) \in \mathbb{R}^{N}$

$$
\tau_{k}(x)=x-\frac{\left\langle x, e_{k}\right\rangle}{\left|e_{k}\right|^{2}} e_{k}=\left(x_{1}, \ldots, x_{k-1}, 0, x_{k+1}, \ldots, x_{N}\right) .
$$

Let $\kappa=\left(\kappa_{1}, \kappa_{2}, \ldots, \kappa_{N}\right) \in \mathbb{C}^{N}$. The associated Dunkl type operators $T_{j}$ for $j=1, \ldots, N$, are given for $x \in \mathbb{R}^{N}$ by

$$
T_{j} f(x)=\partial_{j} f(x)+\sum_{l=1}^{N} \kappa_{l} \frac{f(x)-f\left(\tau_{l}(x)\right)}{\left\langle x, e_{l}\right\rangle}\left\langle e_{k}, e_{l}\right\rangle
$$




$$
=\partial_{j} f(x)+\kappa_{j} \frac{f(x)-f\left(x_{1}, \ldots, x_{j-1}, 0, x_{j+1}, \ldots, x_{N}\right)}{x_{j}} .
$$

These operators form a commuting system. The generalized Laplacian associated with $T_{j}$ is defined in a natural way as

$$
\Delta_{\kappa}=\sum_{j=1}^{N} T_{j}^{2} .
$$

A straightforward computation yields

$$
\begin{aligned}
\Delta_{\kappa}= & \Delta+2 \sum_{j=1}^{N} \kappa_{j} x_{j}^{-1} \partial_{j} f(x)-\sum_{j=1}^{N}\left(\kappa_{j}^{2}+\kappa_{j}\right) x_{j}^{-1} \partial_{j} f\left(x_{1}, \ldots, x_{j-1}, 0, x_{j+1}, \ldots, x_{N}\right) \\
& +\sum_{j=1}^{N}\left(\kappa_{j}^{2}-\kappa_{j}\right) x_{j}^{-2}\left(f(x)-f\left(x_{1}, \ldots, x_{j-1}, 0, x_{j+1}, \ldots, x_{N}\right)\right) .
\end{aligned}
$$

This operator will play in our context a similar role to that of the Euclidean Laplacian in the classical harmonic analysis. Obviously, the trivial choice of the multiplicity function $\kappa=0$, reduces our situation to the analysis related to the classical Laplacian $\Delta$.

Let $\kappa=\left(\kappa_{1}, \ldots, \kappa_{N}\right) \in(0, \infty)^{N}$. For $x, \lambda \in \mathbb{R}^{N}$, we consider the function $M_{\kappa}(\lambda, x)$ which is given as the tensor products

$$
M_{\kappa}(\lambda, x)=\prod_{j=1}^{N} M_{\kappa_{j}}\left(i \lambda_{j} x_{j}\right) .
$$

Theorem 6. For $\lambda=\left(\lambda_{1}, \ldots, \lambda_{N}\right) \in \mathbb{C}^{N}$, the function $M_{\kappa}(\lambda, x)$ is the unique analytic solution of the system

$$
T_{\xi} u(x)=i\langle\lambda, \xi\rangle u(x), \quad u(0)=1, \quad \forall \xi \in \mathbb{C}^{N} .
$$

\subsection{Dunkl-type operators associated to an orthogonal subsystem in a root system of type $A_{N-1}$}

Let $R$ be a root system of type $A_{N-1}$

$$
R=\left\{ \pm\left(e_{i}-e_{j}\right), 1 \leq i<j \leq N\right\} .
$$

Define a positive orthogonal subsystem $R^{\prime}=\left\{\alpha_{1}, \ldots, \alpha_{[N / 2]}\right\}$ of $R$ by setting:

$$
\alpha_{i}=e_{2 i-1}-e_{2 i}, \quad i=1, \ldots,[N / 2] .
$$

We denote by $\tau_{j}$ (for each $j$ from 1 to $[N / 2]$ ) the orthogonal projection onto the hyperplane perpendicular to $\alpha_{j}$, that is to say for every $x=\left(x_{1}, \ldots, x_{N}\right) \in \mathbb{R}^{N}$

$$
\tau_{i} x=\left(x_{1}, \ldots, \bar{x}_{2 i-1}, \bar{x}_{2 i}, \ldots, x_{N}\right),
$$

where $\bar{x}_{2 i-1}=\bar{x}_{2 i}=\frac{1}{2}\left(x_{2 i-1}+x_{2 i}\right), i=1, \ldots,[N / 2]$. The vector $\xi \in \mathbb{R}^{N}$ can be decomposed uniquely in the form

$$
\xi=\sum_{i=1}^{[N / 2]} \xi_{i}\left(e_{2 i-1}-e_{2 i}\right)+\widehat{\xi}
$$

where $\widehat{\xi}$ is an orthogonal vector to the linear space generated by $R^{\prime}=\left\{\alpha_{1}, \ldots, \alpha_{[N / 2]}\right\}$. 
A straightforward computation shows that the operator $T_{\xi}\left(\xi \in \mathbb{R}^{N}\right)$ associated with $R^{\prime}$ and the multiplicity parameters $\left(\kappa_{1}, \ldots, \kappa_{[N / 2]}\right)$ has the following decomposition

$$
T_{\xi}=\sum_{i=1}^{[N / 2]} \xi_{i} T_{\alpha_{i}}+\partial_{\widehat{\xi}}=\sum_{i=1}^{2[N / 2]}(-1)^{i+1} \xi_{\left[\frac{i+1}{2}\right]} T_{i}+\partial_{\widehat{\xi}},
$$

where

$$
T_{i}=\partial_{i}-(-1)^{i} \kappa_{\left[\frac{i+1}{2}\right]} \rho_{\left[\frac{i+1}{2}\right]}, \quad i=1, \ldots, 2[N / 2],
$$

and

$$
\left(\rho_{i} f\right)(x)=\frac{f(x)-f\left(\tau_{i} x\right)}{x_{2 i-1}-x_{2 i}} .
$$

The intertwining operator (3) becomes

$$
\chi_{\kappa}(f)(x)=\frac{1}{\Gamma(\kappa)} \int_{[0,1]^{n}} f(h(t, x)) w(t) \mathrm{d} t,
$$

where

$$
h(t, x)=x+\sum_{i=1}^{[N / 2]} \frac{t_{i}-1}{2}\left(x_{2 i-1}-x_{2 i}\right)\left(e_{2 i-1}-e_{2 i}\right) .
$$

Proposition 5. Let $\lambda=\left(\lambda_{1}, \ldots, \lambda_{N}\right) \in \mathbb{C}^{N}$, and $\kappa=\left(\kappa_{1}, \ldots, \kappa_{[N / 2]}\right) \in(0, \infty)^{[N / 2]}$. The following eigenvalue problem

$$
T_{\xi} f=i\langle\lambda, \xi\rangle f, \quad f(0)=1, \quad \forall \xi \in \mathbb{C}^{N},
$$

has a unique analytic solution $M_{\kappa}(\lambda, x)$ given by

$$
M_{\kappa}(\lambda, x)=e^{i\langle\lambda, h(0, x)\rangle} \prod_{j=1}^{[N / 2]} M_{\kappa_{j}}\left(\frac{i}{2}\left(\lambda_{2 j-1}-\lambda_{2 j}\right)\left(x_{2 j-1}-x_{2 j}\right)\right) .
$$

Proof. According to Theorem 2, $\chi_{\kappa}$ is an intertwining operator between $T_{\xi}$ and $\partial_{\xi}$. So, the function $\chi_{\kappa}\left(e^{i\langle\lambda,\rangle}\right)$ is the unique $C^{\infty}$-solution of problem (11).

Since we can write

$$
\langle\lambda, h(t, x)\rangle=\langle\lambda, h(0, x)\rangle+\sum_{j=1}^{[N / 2]} \frac{t_{j}}{2}\left(\lambda_{2 j-1}-\lambda_{2 j}\right)\left(x_{2 j-1}-x_{2 j}\right),
$$

we are lead to

$$
\begin{aligned}
M_{\kappa}(\lambda, x) & =\frac{e^{i\langle\lambda, h(0, x)\rangle}}{\Gamma(\kappa)} \int_{[0,1][N / 2]} e^{\frac{i}{2} \sum_{j=1}^{[N / 2]} t_{j}\left(\lambda_{2 j-1}-\lambda_{2 j}\right)\left(x_{2 j-1}-x_{2 j}\right)} w(t) \mathrm{d} t \\
& =e^{i\langle\lambda, h(0, x)\rangle} \prod_{j=1}^{[N / 2]} \frac{1}{\Gamma\left(\kappa_{j}\right)} \int_{[0,1]} e^{\frac{i}{2}\left(\lambda_{2 j-1}-\lambda_{2 j}\right)\left(x_{2 j-1}-x_{2 j}\right)}\left(1-t_{j}\right)^{\kappa_{j}-1} \mathrm{~d} t_{j} .
\end{aligned}
$$

If we now use (7) and (8) we get

$$
M_{\kappa}(\lambda, x)=e^{i\langle\lambda, h(0, x)\rangle} \prod_{j=1}^{[N / 2]} M_{\kappa_{j}}\left(\frac{i}{2}\left(\lambda_{2 j-1}-\lambda_{2 j}\right)\left(x_{2 j-1}-x_{2 j}\right)\right) .
$$




\subsection{Dunkl-type operators associated to orthogonal subsystem in root system of type $B_{N}$}

Throughout this subsection $R$ is a root system of type $B_{N}$ which is given by

$$
R=\left\{ \pm e_{i} \pm e_{j}, 1 \leq i<j \leq N ; \pm e_{i} 1 \leq i \leq N\right\},
$$

and $R^{\prime}$ is a positive orthogonal subsystem $R^{\prime}$ in the root system $R$ given by

$$
R^{\prime}=\left\{\alpha_{i}^{ \pm}=e_{2 i-1} \pm e_{2 i}, 1 \leq i \leq[N / 2]\right\} .
$$

Denote by $\tau_{i}^{ \pm}$(for each $i$ from 1 to $[N / 2]$ ) the orthogonal projection onto the hyperplane perpendicular to $\alpha_{i}^{ \pm}$, that is to say for every $x=\left(x_{1}, \ldots, x_{N}\right) \in \mathbb{R}^{N}$

$$
\tau_{i}^{ \pm} x=\left(x_{1}, \ldots, \bar{x}_{2 i-1}^{ \pm}, \bar{x}_{2 i}^{ \pm}, \ldots, x_{N}\right),
$$

where $\bar{x}_{2 i-1}^{ \pm}=\bar{x}_{2 i}^{ \pm}=\frac{1}{2}\left(x_{2 i-1} \pm x_{2 i}\right)$. In this case, the Dunkl type operator $T_{\xi}$ associated with $R^{\prime}$ and the multiplicity parameters $\left(\kappa_{1}^{ \pm}, \ldots, \kappa_{[N / 2]}^{ \pm}\right)$takes the form

$$
\left(T_{\xi} f\right)(x)=\partial_{\xi} f(x)+\sum_{j=1}^{[N / 2]} \kappa_{j}^{-}\left\langle\alpha_{j}^{-}, \xi\right\rangle \frac{f(x)-f\left(\tau_{j}^{-} x\right)}{\left\langle x, \alpha_{j}^{-}\right\rangle}+\kappa_{j}^{+}\left\langle\alpha_{j}^{+}, \xi\right\rangle \frac{f(x)-f\left(\tau_{j}^{+} x\right)}{\left\langle x, \alpha_{j}^{+}\right\rangle} .
$$

In particular, for $i=1, \ldots, 2[N / 2]$ we have

$$
T_{i}=\partial_{i}-(-1)^{i} \kappa_{\left[\frac{i+1}{2}\right]}^{-} \rho_{\left[\frac{i+1}{2}\right]}^{-}+\kappa_{\left[\frac{i+1}{2}\right]}^{+} \rho_{\left[\frac{i+1}{2}\right]}^{+} .
$$

where

$$
\left(\rho_{i}^{ \pm} f\right)(x)=\frac{f(x)-f\left(\tau_{i}^{ \pm} x\right)}{x_{2 i-1} \pm x_{2 i}} .
$$

The operator $T_{\xi}$ has also the following decomposition

$$
T_{\xi}=\sum_{i=1}^{2[N / 2]}\left(\xi_{\left[\frac{i+1}{2}\right]}^{+}+(-1)^{i+1} \xi_{\left[\frac{i+1}{2}\right]}^{-}\right) T_{i}+\varepsilon \xi_{N} \partial_{N}
$$

where

$$
\xi=\sum_{i=1}^{[N / 2]} \xi_{i}^{+} \alpha_{i}^{+}+\xi_{i}^{-} \alpha_{i}^{-}+\varepsilon \xi_{N} e_{N}, \quad \text { and } \quad \varepsilon= \begin{cases}1 & \text { if } N \text { is odd } \\ 0 & \text { if } N \text { is even }\end{cases}
$$

Proposition 6. Let $\lambda=\left(\lambda_{1}, \ldots, \lambda_{N}\right) \in \mathbb{C}^{N}$ and

$$
\kappa=\left(\kappa^{+}, \ldots, \kappa_{[N / 2]}^{+}, \kappa^{-}, \ldots, \kappa_{[N / 2]}^{-}\right) \in(0, \infty)^{2[N / 2]} .
$$

The following eigenvalue problem

$$
T_{\xi} f=i\langle\lambda, \xi\rangle f, \quad f(0)=1, \quad \forall \xi \in \mathbb{C}^{N},
$$

has a unique analytic $M_{\kappa}(\lambda, x)$ given by

$$
\begin{aligned}
M_{\kappa}(\lambda, x)= & e^{i\langle\lambda, h(0, x)\rangle} \prod_{j=1}^{[N / 2]} M_{\kappa_{j}^{-}}\left(\frac{i}{2}\left(\lambda_{2 j-1}-\lambda_{2 j}\right)\left(x_{2 j-1}-x_{2 j}\right)\right) \\
& \times M_{\kappa_{j}^{+}}\left(\frac{i}{2}\left(\lambda_{2 j-1}+\lambda_{2 j}\right)\left(x_{2 j-1}+x_{2 j}\right)\right) .
\end{aligned}
$$




\section{Acknowledgements}

This research is supported by NPST Program of King Saud University, project number 10MAT1293-02. I would like to thank the editor and the anonymous referees for their helpful comments and remarks.

\section{References}

[1] Bouzeffour F., Special functions associated with complex reflection groups, Ramanujan J., to appear.

[2] Cherednik I., Double affine Hecke algebras, Knizhnik-Zamolodchikov equations, and Macdonald's operators, Int. Math. Res. Not. (1992), 171-180.

[3] Dunkl C.F., Reflection groups and orthogonal polynomials on the sphere, Math. Z. 197 (1988), 33-60.

[4] Dunkl C.F., Differential-difference operators associated to reflection groups, Trans. Amer. Math. Soc. 311 (1989), 167-183.

[5] Dunkl C.F., Opdam E.M., Dunkl operators for complex reflection groups, Proc. London Math. Soc. 86 (2003), 70-108, math.RT/0108185.

[6] Dunkl C.F., Xu Y., Orthogonal polynomials of several variables, Encyclopedia of Mathematics and its Applications, Vol. 81, Cambridge University Press, Cambridge, 2001.

[7] Heckman G.J., An elementary approach to the hypergeometric shift operators of Opdam, Invent. Math. 103 (1991), 341-350.

[8] Heckman G.J., Dunkl operators, Astérisque 245 (1997), Exp. No. 828, 4, 223-246.

[9] Humphreys J.E., Reflection groups and Coxeter groups, Cambridge Studies in Advanced Mathematics, Vol. 29, Cambridge University Press, Cambridge, 1990.

[10] Kober H., On fractional integrals and derivatives, Quart. J. Math., Oxford Ser. 11 (1940), $193-211$.

[11] Koornwinder T.H., Bouzeffour F., Nonsymmetric Askey-Wilson polynomials as vector-valued polynomials, Appl. Anal. 90 (2011), 731-746, arXiv:1006.1140.

[12] Luchko Y., Trujillo J.J., Caputo-type modification of the Erdélyi-Kober fractional derivative, Fract. Calc. Appl. Anal. 10 (2007), 249-267.

[13] Macdonald I.G., Affine Hecke algebras and orthogonal polynomials, Cambridge Tracts in Mathematics, Vol. 157, Cambridge University Press, Cambridge, 2003.

[14] Opdam E.M., Dunkl operators, Bessel functions and the discriminant of a finite Coxeter group, Compositio Math. 85 (1993), 333-373.

[15] Temme N.M., Special functions. An introduction to the classical functions of mathematical physics, $A$ WileyInterscience Publication, John Wiley \& Sons Inc., New York, 1996. 